\title{
Dynamic Modeling of a Central Receiver CSP system in Modelica
}

\author{
Johan Edman $^{1} \quad$ Johan Windahl $^{2}$ \\ ${ }^{1}$ Department of Energy Sciences, F. Eng., Lund University, Sweden, edman. jle@gmail.com \\ ${ }^{2}$ Modelon AB, Ideon Science Park, Lund, Sweden, johan.windahl@modelon.com
}

\begin{abstract}
A dynamic model of the Solar Two test facililty has been implemented in Modelica. The model consists of a set of Central Receiver specific CSP components, along with a Rankine cycle to form a complete system. Main components include models of a sun, heliostat field, receiver, storage tank and a Rankine cycle including a steam generator. The components and the full system were tested in a series of simulations - both dynamically and during steady state conditions - and the results were compared to data from the reference system. The dynamic behavior of the models aligned with expectations, although time constants could not be evaluated due to lack of dynamic reference data. The steady state characteristics were adequate for most models, although some complementary work needs to be done on the Receiver model.

Keywords: Modelica, Dymola, Dynamic modeling, Concentrated Solar Power, Central Receiver, Solar Salt, ThermalPower library
\end{abstract}

\section{Introduction}

Due to an increasing energy demand of a growing world population with an increasing consumption of technology, the interest renewable energy is ever increasing. This is further reinforced by a heightened awareness of the impact of the exploitation of non-renewable energy sources on local environments and global climate.

Furthermore, fossil fuels are increasingly being subjected to scrutiny. Reasons for this include the impending threat of global peak oil, i.e. the point when the extraction rate of oil can no longer meet the consumption rate, and the allocation of oil and other fossil resources to instable regions of the world. This has kindled the interest in developing new, local means of power production to minimize the dependence on foreign resources. A promising branch of renewable energy production is concentrated solar power (CSP).

Concentrated Solar Power refers to thermal power systems which use the sun as their primary heat source. The underlying principle behind CSP systems is that the solar radiation incident on Earth is basically collimated and thus can be focused. To produce the heat flux required to maintain an adequate working temperature for efficient operation of a thermal power system the sunlight has to be concentrated several orders of magnitude.

There are several different types of CSP systems, but they all consist of the same elemental components. All CSP systems have a set of Sun-tracking reflectors which concentrate the sunlight onto an absorber. The absorbed power is converted into heat which is either converted directly into electricity using a heat engine or transported to a conventional thermal power cycle via a Heat Transfer Fluid (HTF). Systems using an HTF can often be combined with a heat storage system and/or an auxiliary gas turbine, enabling power generation during insufficient weather conditions and throughout the night.

The Central Receiver system (CRS), which is modelled in this project, uses numerous large mirrors, called Heliostats, which track the movement of the Sun over two axes. Heliostats focus the sunlight from a vast area onto a Receiver located on top of a tower. The HTF is heated in the receiver and then transported to the thermal power cycle, located by the foot of the tower. A sketch of the Solar Two system is presented in Figure 1.

This work is conducted in collaboration with Modelon $\mathrm{AB}$, Sweden, which specializes in physical modeling of dynamic systems using Modelica. The models developed are largely based on the various model libraries in Modelons portfolio, especially the ThermalPower library, the LiquidCooling library and the Modelon Standard library.

This article describes the authors' process of modeling a CRS based on a reference system, and validating the

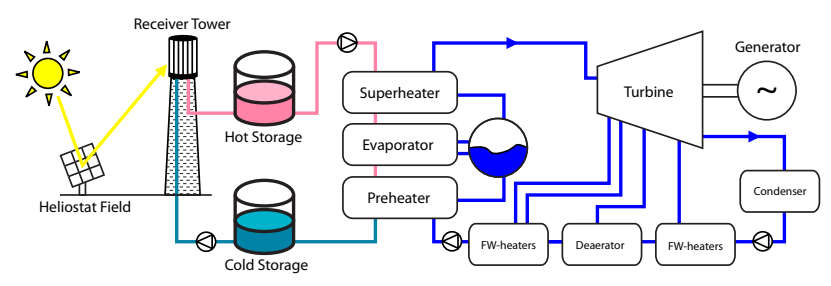

Figure 1. A sketch of the Solar Two CRS system. 
model by conducting simulations. Section 2 describes the reference system, and the properties of the different components. In section 3 the equations governing the behavior of the components are stated, along with the property values used to configure the system model to match the reference system. Section 4 describes the simulations conducted to validate the models, and section 5 lists the simulation results. Finally, in section 6 a discussion of the results is given, along with conclusions and future work.

\section{Reference system}

A reference system was chosen to which the test results could be compared. It can be divided into two parts: The sun including weather conditions and the power plant.

\subsection{Sun}

Reference data for solar properties is taken from measured data collected at Planta Solar de Almeria, Spain (GeoModel Solar, 2014). Important properties are the direct normal insolation (DNI), which describes the solar flux intensity, the solar azimuth $\left(\gamma_{s}\right)$ and elevation angle $\left(\alpha_{s}\right)$, which describe the solar position in the sky.

\subsection{Power Plant}

As most operating systems are commercially owned they do not publish much data. Therefore, an old governmentfunded test plant from the 1990's was chosen as reference system; the Solar Two project in California, USA. The Solar Two project was built to test and gain operating experience in using molten salt as HTF. It was an important predecessor of large, commercial plants such as the Gemasolar plant, formerly called Solar Tres, in Seville, Spain.

Reference data for Solar Two is taken from public reports (such as Pacheco, 2002) by Sandia National Laboratories, which was one of the main actors of the project.

Solar Two consists of a central tower in a surround heliostat field, an external cylinder receiver, a direct heat storage system and an electrical power generation system (EPGS) consisting of a steam generator producing steam for a 12.5 MW Rankine cycle. The HTF is a molten nitrate salt solution.

Heliostat Field The heliostat field is a north-biased surround field. It consist of 1818 relatively small healiostats à $39 \mathrm{~m}^{2}$ and 108 larger heliostats à $95 \mathrm{~m}^{2}$, adding up to a total reflective area of $82,700 \mathrm{~m}^{2}$.

Receiver The receiver consists of 24 panels arranged in a cylinder. Each panel contains 32 thin tubes through which the HTF flows. The exterior sides of the panels are coated with black Pyromark paint, designed to have a high absorptivity (95\%) and thermal endurance.

During normal operation the $\mathrm{HTF}$ enters at $290^{\circ} \mathrm{C}$, and the flow is regulated so the exit temperature is kept constant at $565^{\circ} \mathrm{C}$.

Storage System The direct heat storage system consists of two insulated storage tanks, one for hot HTF $\left(565^{\circ} \mathrm{C}\right)$ and one for cold $\left(290^{\circ} \mathrm{C}\right)$.

The salt flow through the receiver (cold tank $\rightarrow$ hot tank) and the flow through the steam generator (hot tank $\rightarrow$ cold tank) are independent of each other as long as none of the storage tanks are completely empty, and can be regulated separately.

Heat Transfer/Storage Medium The medium commonly referred to as Solar Salt is used both as heat transfer and as heat storage medium. It is a mixture of $60 \%$ sodium nitrate $\left(\mathrm{NaNO}_{3}\right)$ and $40 \%$ potassium nitrate $\left(K_{N} O_{3}\right)$. Solar salt has a high heat capacity and a low vapor pressure. It starts to crystallize at $240^{\circ} \mathrm{C}$ and is completely solid at $220^{\circ} \mathrm{C}$ (Ferri et al., 2008). Consequently, the salt has to be kept above these temperatures or it may cause major damage to parts of the system.

Steam Generator The steam generator consists of a preheater, a kettle evaporator and a superheater. The preheater and superheater are U-tube, straight shelled heat exchangers.

During normal operation, feedwater enter the preheater at $260^{\circ} \mathrm{C}$ and $100 \mathrm{bar}$. The preheater heat the feedwater to near a saturation temperature at $311^{\circ} \mathrm{C}$, the evaporator produces steam at $311^{\circ} \mathrm{C}$ and finally the steam is heated in the superheater to $535^{\circ} \mathrm{C}$.

The steam turbine, described in the next section, was salvaged from an old project and was not dimensioned to handle an inlet temperature of $535^{\circ} \mathrm{C}$. Consequently, the steam had to be attemperated using feedwater to bring the temperature down to $510^{\circ} \mathrm{C}$. The reason for producing steam at a higher temperature than the turbine could handle was to demonstrate the potential of the solar salt technology.

Rankine Cycle The power cycle is a non-reheat regenerative Rankine cycle using a train configuration with four extraction points. The first and second extraction points were fed to two high pressure feedwater heaters, the third point to a deaerator and the last point to a low pressure heater.

The cycle had a rated gross electrical output of 12.5 MW at 0.084 bar condenser pressure, a nominal steam mass flow rate of $13.9 \mathrm{~kg} / \mathrm{s}$ and inlet conditions as described above, i.e. $510^{\circ} \mathrm{C}$ and $100 \mathrm{bar}$.

The steam turbine was salvaged from the Solar One project (the predecessor of Solar Two) which was conducted in the 1980's, and refurbished. 


\section{Implementation}

Six major components, along with important subcomponents, were implemented: The Sun model, the Heliostat Field model, the Receiver model, the Storage Tank model, the Steam Generator model and the Rankine Cycle model. Components which are affected by ambient factors - such as ambient temperature, pressure and wind speed - contain an outer component called Weather Conditions, which allows these parameters to be set globally.

\subsection{Sun}

The Sun model provides input values to the Heliostat Field model. Outputs are DNI, elevation angle $\alpha_{s}$ and the azimuth angle $\gamma_{s}$. To calculate the elevation and azimuth angles the hour angle $\omega$ and the declination angle $\delta$ needs first to be calculated.

For a given point in time (day number $n$ and solar time $h \mathrm{~h}: \mathrm{mm}: \mathrm{ss}$ ) and latitude $\lambda$ the other angles are calculated as follows, here in degrees (Duffie et al., 2013):

$$
\begin{aligned}
\omega & =15(h h-12)+m m / 4+s s / 240 \\
\delta & =23.45 \sin (360(284+n) / 365) \\
\alpha_{s} & =\sin ^{-1}(\cos \delta \cos \omega \cos \lambda+\sin \delta \sin \lambda) \\
\gamma_{s} & =\operatorname{sgn}(\omega)\left|\cos ^{-1}\left(\frac{\cos \delta \cos \omega \sin \lambda-\sin \delta \cos \lambda}{\cos \alpha_{s}}\right)\right|
\end{aligned}
$$

(4) The receiver model converts incoming insolation into

\subsection{Heliostat Field}

The Heliostat Field model converts inputs from the Sun model into total insolation onto the receiver. The implementation can be summarized with the following formula:

$$
I=\mathrm{DNI} \cdot E_{\eta}\left(\alpha_{s}, \gamma_{s}\right) \cdot A \cdot \eta_{r} \cdot \eta_{c} \cdot \alpha_{r e c}
$$

where

$$
\begin{aligned}
I & =\text { insolation to receiver } \quad[W] \\
E_{\eta}\left(\alpha_{s}, \gamma_{s}\right) & =\text { Efficiency matrix } \\
A & =\text { Total reflective area } \quad\left[\mathrm{m}^{2}\right]
\end{aligned}
$$
heat and transfers it to the HTF. It also accounts for ambient heat losses through radiation and convection. The implementation consists of three main subcomponents; a surface model, a wall model and a pipe model. Each of the subcomponents is discretized into $n$ segments. The Receiver model is displayed in Figure 2.

A few simplifications have been made in this implementation:

and

$$
\begin{aligned}
\eta_{r} & = \begin{cases}\text { reflectivity } & \text { if not inclRefl } \\
1.0 & \text { else }\end{cases} \\
\eta_{c} & = \begin{cases}\text { cleanliness } & \text { if not inclclean } \\
1.0 & \text { else }\end{cases} \\
\alpha_{\text {rec }} & = \begin{cases}1 / \text { rec. abs. } & \text { if inclRecAbs } \\
1.0 & \text { else }\end{cases}
\end{aligned}
$$

The efficiency of the field is determined by an externally provided matrix. The efficiency matrix is inserted into a lookup table which takes the elevation and azimuth angles as input and outputs an interpolated efficiency value.

The following simplifications are used:

1. The entire heliostat field is always focused on the receiver, no capability to defocus the field is implemented.

2. The output is the total power reflected towards the receiver, no information on the distribution of the insolation from different directions or along the height or the receiver is provided.

\subsection{Receiver}

1. The emissivity of the receiver surface is independent of the surface temperature (Gray body assumption).

2. The convective heat loss in each surface node is calculated as an estimate of the mean convective heat loss for a cylinder in cross flow with a surface temperature equal to the node temperature (see the Surface model section below).

3. Conduction heat losses are neglected.

4. Internal components used during start-up and shutdown sequences are left out for simplicity. 


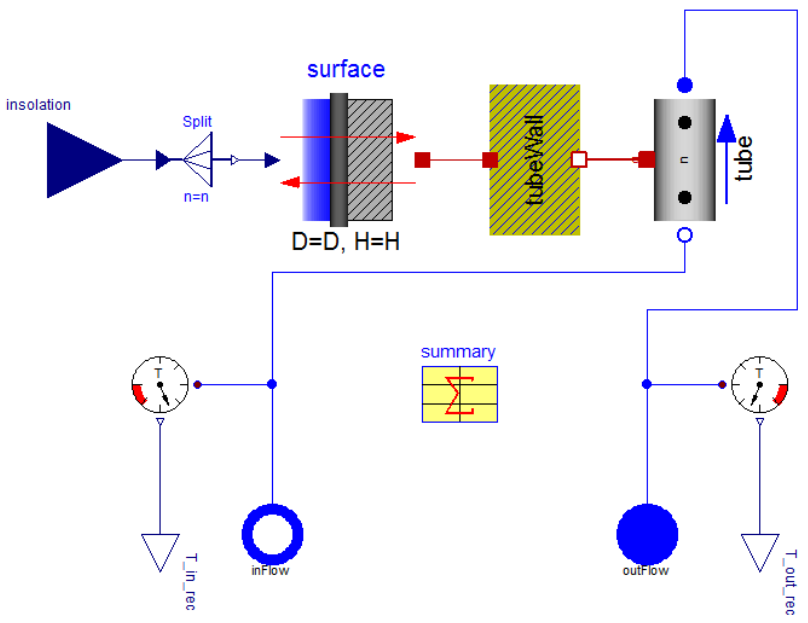

Figure 2. The Receiver model.

\subsubsection{Surface model}

The surface model is a subcomponent which calculates the heat exchanged between the receiver wall and its ambient surroundings. Insolation is converted to heat, heat losses through radiation and convection are subtracted and the net heat is transferred through the heat port.

The model is described by following equations:

$$
\begin{aligned}
\dot{Q}_{\text {net }} & =\dot{Q}_{a b s}-\dot{Q}_{\text {rad }}-\dot{Q}_{\text {conv }} & & {[W] } \\
\dot{Q}_{a b s} & =\alpha I & & {[W] } \\
\dot{Q}_{\text {rad }} & =\varepsilon \sigma A\left(T_{\text {wall }}^{4}-T_{\text {sky }}^{4}\right) & & {[W] } \\
\dot{Q}_{\text {conv }} & =h_{c} A\left(T_{\text {wall }}-T_{\text {amb }}\right) & & {[W] }
\end{aligned}
$$

where

$$
\begin{array}{rlr}
I & =\text { insolation } & \\
\alpha & =\text { surface absorptivity } & \\
\varepsilon & =\text { surface emissivity } & \\
\sigma & =\text { Stefan-Boltzmann constant } & {\left[W / m^{2} K^{4}\right]} \\
A & =\text { surface area } & {\left[m^{2}\right]} \\
h_{c} & =\text { convective heat transfer coefficient } & {\left[W / m^{2} K\right]}
\end{array}
$$

The wall temperature $T_{\text {wall }}$ is taken from the heat port, the ambient temperature $T_{a m b}$ is taken from the weather conditions model and the sky temperature $T_{s k y}$ is estimated as $T_{a m b}-8$ (Forristal, 2013).

The convective heat transfer coefficient $h_{c}$ is calculated as the sum of forced and natural convection.

$$
h_{c}=h_{c n}+h_{c f}
$$

For the natural convective heat transfer coefficient, $h_{c n}$, a correlation for the Nusselt number for a vertical plate is used (Churchill et al., 1975). The formula is modified to better fit vertical cylinders by adding a second term (Fujii et al., 1970).

$$
\begin{aligned}
h_{c n} & =\frac{N u_{H} k}{H} \\
N u_{H} & =\left(0.825+\frac{0.387\left(G r_{H} P r\right)^{1 / 6}}{\left(1+\left(\frac{0.492}{P r}\right)^{9 / 16}\right)^{8 / 27}}\right)^{2}+0.97 \frac{H}{D}
\end{aligned}
$$

where

$$
\begin{array}{rlr}
H & =\text { height of the receiver } & {[m]} \\
D & =\text { diameter of the receiver } & {[m]} \\
G r_{H} & =\text { Grashof number } & \\
P r & =\text { Prandtl number }
\end{array}
$$

For the forced convective heat transfer coefficient, $h_{c f}$, a correlation for the Nusselt number for a cylinder in cross-flow is used (Churchill et al., 1977):

$$
\begin{aligned}
& h_{c f}=\frac{N u_{D} k}{D} \\
& N u_{D}=0.3+\frac{0.62 R e^{1 / 2} \operatorname{Pr}^{1 / 3}}{\left(1+\left(\frac{0.4}{P r}\right)^{2 / 3}\right)^{1 / 4}}\left(1+\left(\frac{R e}{282000}\right)^{5 / 8}\right)^{4 / 5} \\
& \text { [m] } \\
& R e=\text { Reynolds number }
\end{aligned}
$$

\subsection{Storage Tank}

The storage tank model consists of a two-media volume, a wall and two heat conductors. Heat ports of the volume are connected to a fixed temperature source via the wall model and two conductor models (see the Film Conductor section below). Values for wall properties are given as the lumped properties of the metal wall and the insulation.

\subsubsection{Film Conductor}

The FilmConductor model is created to provide a conductor model with a variable heat transfer area. The heat transfer area is set to depend on the liquid level in the tank. As the conductor model is separate from the wall model, the conduction through the liquid layer closest to the tank wall is modelled. The thermal conductivity is evaluated at the film temperature. It is described by following equations: 


$$
\begin{aligned}
\dot{Q} & =k\left(T_{\text {film }}\right) \cdot A_{\text {heat }} \cdot\left(T_{\text {fluid }}-T_{\text {wall }}\right) \\
T_{\text {film }} & =\frac{T_{\text {wall }}+T_{\text {fluid }}}{2}
\end{aligned}
$$

where

$$
k=\text { Thermal conductivity } \quad[W / m K]
$$

The thickness of the film is given a default value of $0.2 \mathrm{~m}$.

\subsection{Steam Generator}

In the steam generator model, heat from the HTF is transferred to the water in the Rankine cycle in three static HEX models; the preheater, the boiler and the superheater. Steam is produced in a drum model connected to the boiler. The feedwater flow is regulated to keep the drum level constant. Volumes are placed between the flow components to provide numerical stability and thermal inertia. The Steam Generator model is presented in Figure 3.

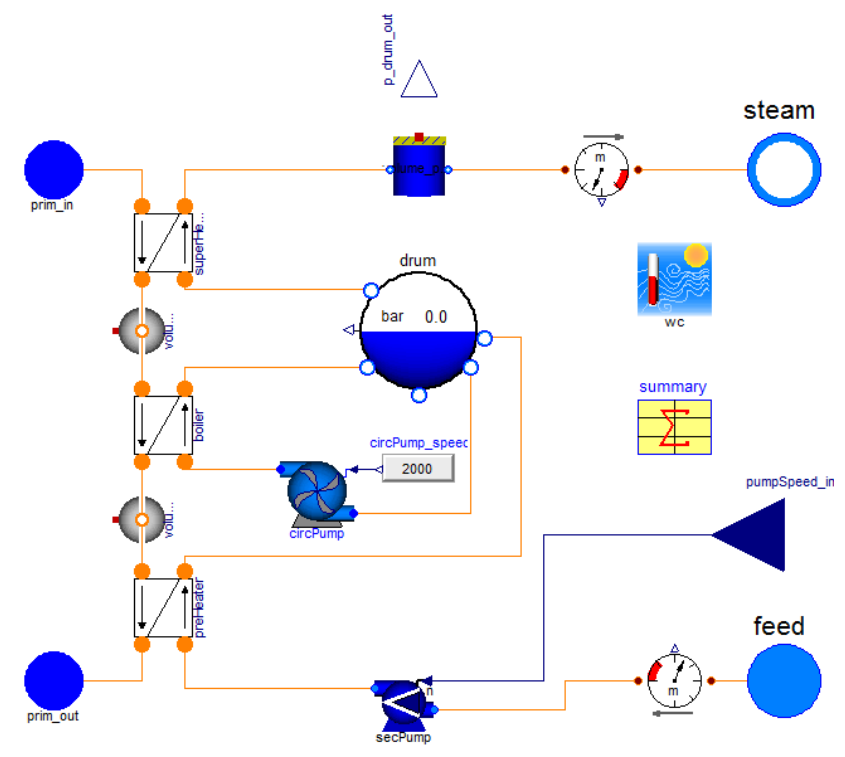

Figure 3. The Steam Generator model.

\subsection{Rankine Cycle}

The Rankine cycle model is a slightly simplified model of the Solar Two steam cycle plant. It consists of four turbine segment, a condenser, a generator (modelled as an efficiency parameter) and three open feedwater heater components. The turbine models are based on Stodola's law. The heater components consist of a deaerator, a pump and a check valve. The pump speed is regulated to keep the liquid level in the deaerator at a constant level.

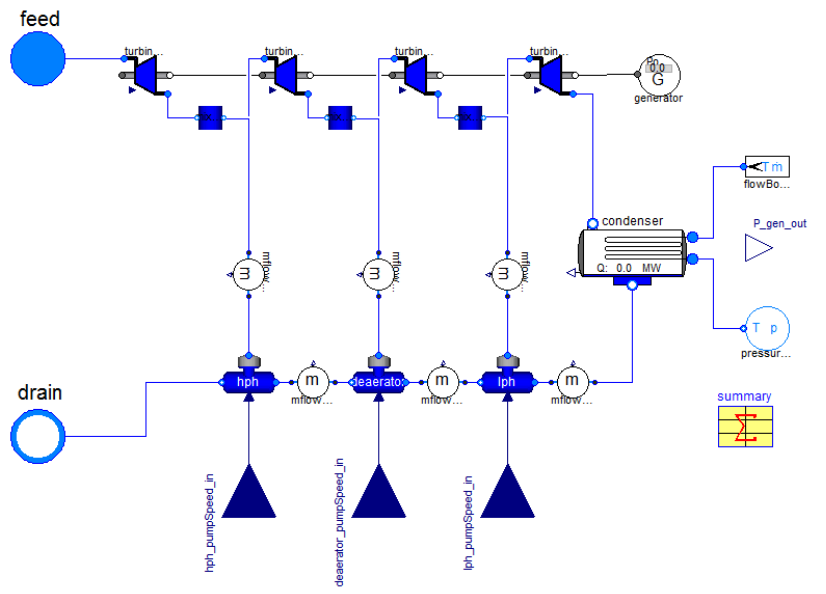

Figure 4. The Rankine model.

Preheaters and turbine segments are connected in a train configuration. Only open feedwater heaters (deaerators) are used as it allows for easy configuration of the setup (Haywood, 1991). The Rankine model is displayed in Figure 4.

\subsection{Configuration of the System model}

In the system model, components are combined and configured according to data from the reference system presented in section 2.2. All salt/water flows are regulated by a master control model.

As no efficiency matrix has been published for the Solar Two heliostat field, a matrix calculated for a similar type of field was modified and used. The matrix is generated by Sandia National Laboratories using an algorithm called DELSOL, written in FORTRAN (Ehrhart et al., 2013). Inputs to DELSOL differ slightly from properties of the Solar Two plant, which will cause an error. However, in the absence of a better approximation this matrix was used.

The heat transfer properties of the storage tanks were set to guess values. No effort was made to fine tune the heat loss from the tanks as its magnitude was several orders smaller than the heat transported through the tanks.

The Rankine model is configured to maximize its efficiency. The efficiency of a non-reheat, regenerative Rankine cycle with only open feedwater heaters is maximized when the enthalpy rise is equal between two adjacent heaters (Haywood, 1991). Knowing the condenser back pressure and final feedwater temperature, the enthalpy in the condenser and high presseure heater can be computed. The intermediate enthalpy levels can then be calculated and the pressures are determined as the saturation pressures with corresponding liquid phase enthalpies.

Setting the isentropic efficiency of each turbine segment to 0.7 , which is reasonable for a turbine of this size 


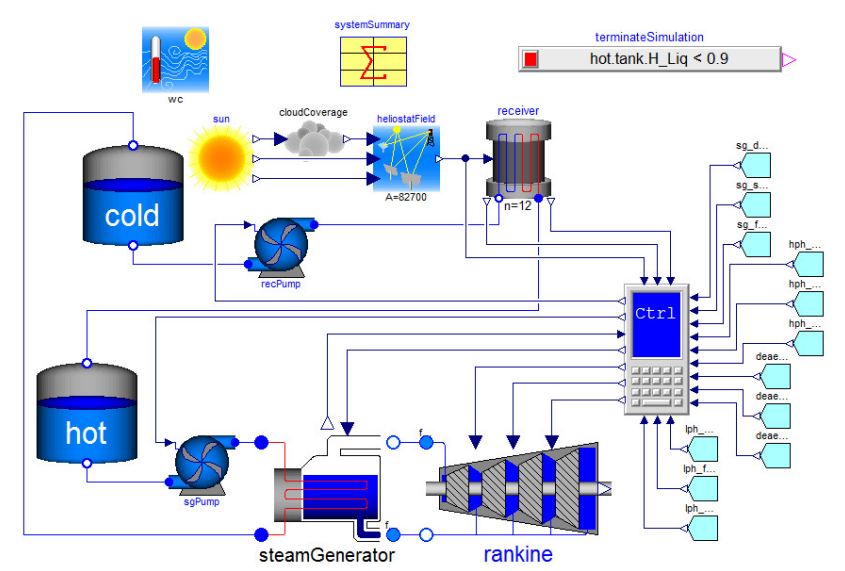

Figure 5. The System model setup.

and age (Haywood, 1991; Thern, 2013), the enthalpy levels in the turbine are iteratively calculated as

$$
h_{i+1}=h_{i}-\eta_{i s}\left(h_{i}-h_{i s}\left(p_{i+1}\right)\right)
$$

starting with the entalpy of the inlet steam.

The final values of pressures and entahlpies at the nominal operating point are presented in Table 1 .

\begin{tabular}{lrrr}
\hline Parameter & $\begin{array}{r}\text { Pressure } \\
{[\mathrm{bar}]}\end{array}$ & $\begin{array}{r}\mathrm{H}, \text { steam } \\
{[\mathrm{kJ} / \mathrm{kg}]}\end{array}$ & $\begin{array}{r}\mathrm{H}, \text { liquid } \\
{[\mathrm{kJ} / \mathrm{kg}]}\end{array}$ \\
\hline Inlet steam & 100.000 & 3464.28 & \\
1st extraction & 46.920 & 3294.26 & 1134.83 \\
2nd extraction & 13.065 & 3051.71 & 815.80 \\
3rd extraction & 1.885 & 2769.13 & 496.76 \\
Condenser & 0.084 & 2435.92 & 177.73 \\
\hline
\end{tabular}

Table 1. Nominal pressure and enthalpy values for the Rankine model.

The full system model is diplayed in Figure 5.

\section{Testing and Simulation}

Each component was tested dynamically and in steady state and results were compared to data from the reference system if it was available. In this section setups from the Receiver and the full system tests are presented. For a further detailed description of the tests, see Edman (2014).

\subsection{Receiver}

Two tests are presented for the receiver; one test of the dynamics and one test measuring the efficiency at steady state conditions.

\subsubsection{Dynamics}

The receiver is initially fed a constant insolation of $35 \mathrm{MW}$ and a salt mass flow rate of $60 \mathrm{~kg} / \mathrm{s}$. At $t=5 \mathrm{~min}$, the insolation is increased to $45 \mathrm{MW}$, and at $t=10 \mathrm{~min}$ the mass flow rate is increased to $80 \mathrm{~kg} / \mathrm{s}$. The temperature of the salt outflow and in the tubewalls, and the total heat loss at the surface are monitored.

\subsubsection{Steady State}

The steady state efficiency of the receiver was tested at different levels of insolation and wind speed. The salt flow was regulated to keep the receiver outflow temperature constant at $565^{\circ} \mathrm{C}$. Once the flow is stabilized the efficiency is calculated as:

$$
\eta_{\text {rec }}=\frac{Q_{\text {net }}}{Q_{\text {inc }}}=\frac{\dot{m} \cdot\left(h_{\text {out }}-h_{\text {in }}\right)}{I}
$$

The input values are presented in Table 2 .

\begin{tabular}{lrc}
\hline Parameter & \multicolumn{2}{c}{ Values } \\
\hline Insolation & $\{48,40,30,20,15\}$ & {$[\mathrm{MW}]$} \\
Wind speed at $76.2 \mathrm{~m}$ & $\{0,2.5,5,7.5,10\}$ & {$[\mathrm{m} / \mathrm{s}]$} \\
\hline
\end{tabular}

Table 2. Parameter values used in the Receiver Steady State test.

\subsection{System}

Two tests are conducted including all parts of the system, one steady state test and one dynamics test. Parameter and initial values are configured according to section 2.2.

\subsubsection{Steady State}

In the steady state test the receiver is fed a constant level of insolation. The mass flow rate of salt through the receiver is regulated to keep the outlet temperature at a constant level of $565^{\circ} \mathrm{C}$. The steam generator is fed with the same salt flow rate as the receiver, thus keeping the salt level in the tanks constant. Once the system has reached steady state, heat flow rates and efficiencies are determined and compared to reference data (Pacheco, 2002).

Four simulations are run with diffent levels of insolation. The input parameters are listed in Table 3.

\begin{tabular}{lcccc}
\hline & Case 1 & Case 2 & Case 3 & Case 4 \\
\hline Time & $12: 00$ & $12: 00$ & $09: 00$ & $09: 00$ \\
Day & 172 & 354 & 172 & 354 \\
\hline
\end{tabular}

Table 3. Times for the System Steady State test.

\subsubsection{Dynamics}

The whole system is simulated over a couple of full day scenarios, Clear and Cloudy. Salt flows are regulated to 
maintain nominal operating conditions. Each simulation is started at $t=06: 00$ as the start-up sequence of the receiver is not modelled. The simulation is terminated automatically when the salt level in the hot tank drops below $0.9 \mathrm{~m}$.

Both simulations are run on day number 172. In the Clear scenario a clear day is simulated. In the Cloudy scenario a cloud appears at $t=11: 00$ and obstructs the insolation for one hour. The energy flow rates in the different parts of the system as well as the tank levels are monitored.

\section{Results}

\subsection{Receiver Dynamics}

Results from the dynamic receiver test are presented in Figure 6. In the first $100 \mathrm{~s}$ the receiver tube walls are heated from their initial value of $290^{\circ} \mathrm{C}$ to their steady state value.

When the insolation is increased at $t=5 \mathrm{~min}$ the wall temperature starts to rise, leading to an increased heat flow rate to the salt flow and thus an increased outflow temperature. When the salt flow rate is increased at $t=10 \mathrm{~min}$ the ouflow temperature drops as a greater amount of salt is heated. The increasing temperature difference along with the increased flow rate leads to an increased heat flow rate to the salt flow, causing the wall temperature to drop.

The rise times of the slopes are approximately equal to the time it takes for one mass unit of salt to pass through the receiver $(\approx 60 \mathrm{~s} @ 60 \mathrm{~kg} / \mathrm{s}$ and $45 \mathrm{~s} @ 80 \mathrm{~kg} / \mathrm{s})$. The smoothening at the end of the slopes is due to the thermal inertia of the tube walls.

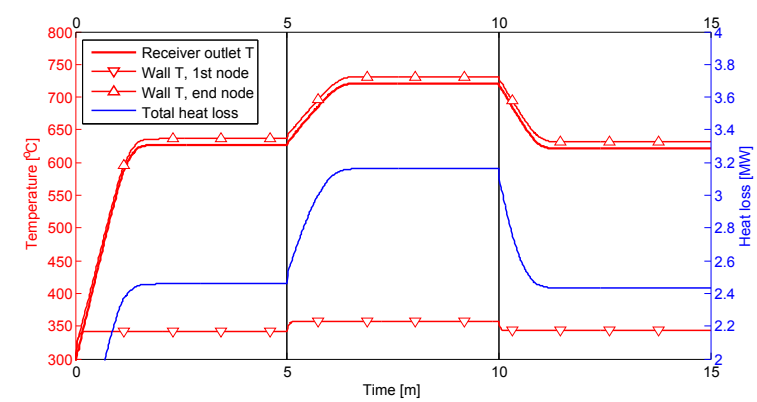

Figure 6. The results from the Receiver dynamics test. Color indicates the correct $y$-axis.

\subsection{Receiver Steady State}

Receiver efficiencies at steady state conditions for different levels of insolation and wind speed are presented in Figure 7.

The efficiency peaks at high insolation and low wind speed and is steadily decreasing with decreasing insola-

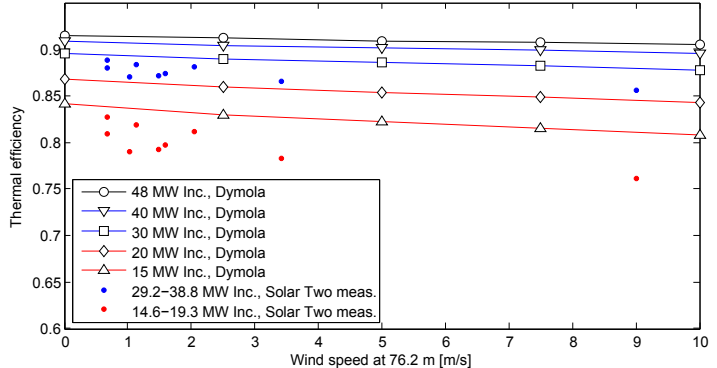

Figure 7. Steady state efficiency of the Receiver model at different levels of insolation and wind speed. The colored markers indicate measured values from the Solar Two project.

tion and increasing wind speed. The blue and red markers in the figure are measured values from the Solar Two project (Pacheco, 2002). Although the behavior of the receiver model is correct the efficiency of the model is generally higher than the measured values from the Solar Two project.

\subsection{System Steady State}

A visual presentation of the first steady state case is presented in Figure 8 . The majority of the losses in the system occur in the heliostat field (optical losses) and in the condenser of the Rankine cycle, and thus these are interesting areas to analyze when developing the system as small improvements may have large impacts.

The power output is higher than the rated power of the Solar Two Turbine $(12.5 \mathrm{MW})$ as the steam is not attemperated. Also, the salt flow is higher than the nominal flow as it regulated by the receiver flow. If the steam generator salt flow was separately regulated, this would correspond to the hot tank salt level rising.

A comparison between the solar specific component efficiencies in the four different cases and measured values from the Solar Two project is presented in Table 4.

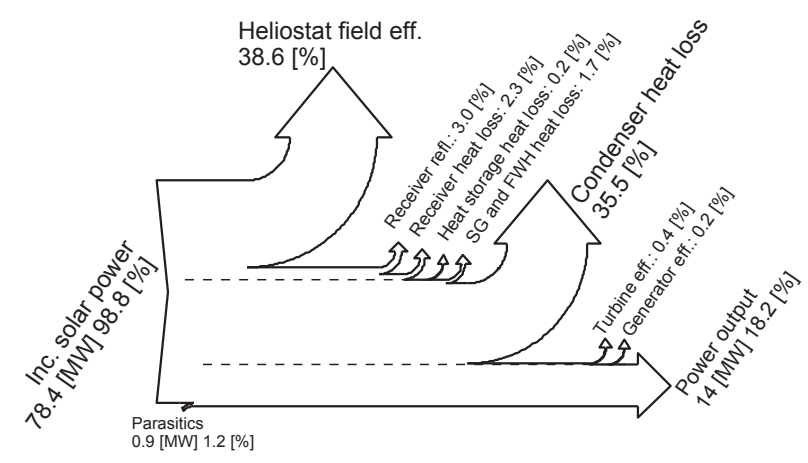

Figure 8. Visualization of the losses from different parts of the system in the System Steady State test. Solid lines are the cloudy scenario and the dashed lines are the clear scenario 


\begin{tabular}{lccccc}
\hline \multicolumn{5}{c}{ Efficiency } \\
\hline & Case 1 & Case 2 & Case 3 & Case 4 & Solar Two \\
HS field & 0.71 & 0.65 & 0.67 & 0.54 & 0.63 \\
Receiver & 0.91 & 0.91 & 0.91 & 0.89 & 0.88 \\
\hline Overall & 0.18 & 0.17 & 0.17 & 0.13 & 0.13 \\
\hline
\end{tabular}

Table 4. Comparison between simulated efficiencies and the measured Solar Two efficiencies(Pacheco, 2002).

The heliostat field and receiver models are in general more efficient than the measured values, resulting in a generally higher overall efficiency. The efficiencies of the other parts of the system did not differ significantly from the measured values.

\subsection{System Dynamics}

Figure 9 shows energy flow rates in different parts of the system (top), and the liquid levels in the tanks (bottom), from both scenarios.

Power production continues virtually undisturbed during the passing of the cloud and well after the insolation sinks below the power input demanded by the power cycle. However, in the cloudy scenario the simulation is stopped earlier as power has been drained from the storage during the passing of the cloud and thus the hot tank empties sooner.

The level in the hot tank sinks in the early hours as the absorbed power by the receiver is lower than the power delivered to the steam generator. Once the absorbed power exceeds the demand the level starts to rise, and continues to do so until the demand is once again higher than the absorbed power. In the Cloudy scenario

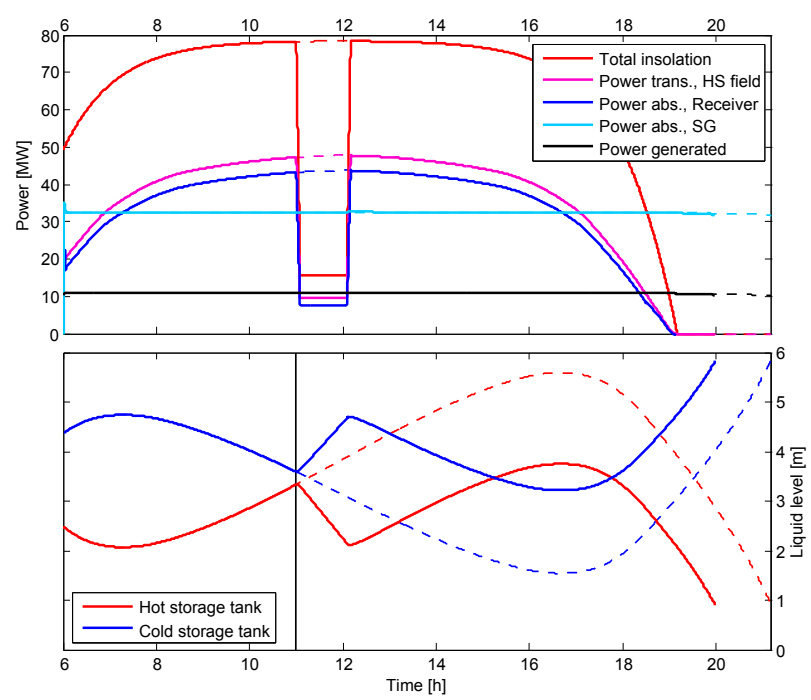

Figure 9. Top: Power transferred in the different parts of the system in the System Dynamics test. Bottom: Variations in tank liquid levels. The dashed lines represent the Clear scenario and the solid lines represent the Cloudy scenario. the hot salt level drops at a constant rate during the passing of the cloud as the incoming flow from the receiver is very low.

\section{Discussion}

As relevant dynamic data from the reference system is scarce, time constants and general appearance of the dynamic results cannot be validated against a reference, only discussed from a theoretical point of view. To get feedback from the reference system the steady state heat loss and efficiency tests were conducted.

\subsection{Evaluation of Results}

\subsubsection{Receiver model}

The dynamical behavior of the receiver is satisfactory. However, there is room for improvement as start-up and shut-down procedures are not modeled.

The steady state efficiency of the receiver model is generally higher than the measured values. A few influential factors can be mentioned:

1. No conduction heat loss has been implemented in the receiver model, see further discussion below.

2. The insolation is always uniformly distributed among the surface nodes in the model.

3. In the Solar Two receiver efficiency measurement, neither inlet nor outlet salt temperatures were stringently kept at their nominal values (Pacheco, 2002).

Conduction is seldom mentioned in conjunction with receiver heat loss and it was assumed that its influence is negligible. This assumption may need to be reevaluated. The difference between simulated and measured efficiency seems to be proportional to the level of insolation, suggesting a constant heat loss factor is missing from the model. This factor could be due to conduction or possible an inaccurate absorptivity of the receiver surface. However, it does not appear to vary significantly with wind speed, which suggests that the convective heat loss is properly modelled.

\subsubsection{System model}

Steady State The overall efficiency of the system model is generally higher than the measured values. Differing factors are mainly the heliostat field, the receiver (already discussed) and parasitics.

The efficiency of the heliostat field model is higher than the Solar Two HS field. As this efficiency is determined by the efficiency matrix, using a correct matrix would eliminate this error. As the heliostat field is one of the major power sinks in the system, a correct description of its properties is important. 
Parasitics have not been thoroughly studied in this project and many of the parasitic components of the real system have been omitted from the models. Therefore, the parasitic efficiency was higher in the model.

Dynamics The dynamic behavior of the system aligned with expectations and demonstrates the benefit of using a direct storage system. The power production is virtually undisturbed by the passing of the cloud. It is clear that the capacity of this particular storage system is not very large, as the liquid level sinks quickly when the incoming flow of hot salt is disrupted. A commersial system with a larger storage capacity would be able to handle even more perturbances. However, during particularly bad conditions an auxilliary heat source would be needed.

\subsection{Conclusions}

The dynamic behavior of the receiver model is properly modelled to what extent it is possible to verify. Responses to sudden changes in the input parameters are gradual and the model stabilizes at resonable values. However, it is hard to evaluate time constants of the system as most data from the reference system is given as efficiencies and average values, and dynamic data is very scarce.

The two components which are unique for the CRS system are the heliostat field and the receiver. The heliostat field model utilizes the data which is most commonly given for a real field, but more detailed data is needed to improve the model. The receiver model is more efficient than the real system, which is most likely due to the lack of conduction losses and possibly a faulty absorptivity value. More detailed inputs from the heliostat field and the ambient conditions would also improve the model.

The EPGS models work properly, which validates the usefulness of the Modelon ThermalPower library as most components are taken from there.

All models are generic and rescalable. However, the Rankine model has to be modified if a different configuration is used, e.g. with more extraction points; with closed feedwater heaters or with a reheat configuration.

Dymola and the Modelica language, are powerful tools for modeling the thermohydraulic parts of the system, i.e. the solar loop and the power cycle. For detailed modeling of complex optical systems such as the heliostat field, an optical simulation tool would be needed.

\subsection{Future work}

More detail could be added to increase accuracy and allow for more specific properties to be studied.
1. Heliostat/Receiver models: More detailed information about the insolation patterns onto the receiver are needed to increase the model accuracy.

2. HTF model: To be able to model filling and draining of receiver and pipes. During start-up and shutdown sequences the HTF medium must be able to handle a mixture of solar salt and air.

3. Storage Tank model: Stratification and auxilliary heating of the storage tanks have not been modelled.

4. Steam Generator model: Steam attemperation with feedwater should be included in the steam generator model. Also, changing the static heat exchanger models to dynamic ones should be considered.

5. Rankine model: A more generic Rankine model could be implemented.

\section{References}

W. Churchill, H. H. S. Chu, "Correlating equations for laminar and turbulent free convection from vertical plate," Int. J. Heat. Mass. Tran. 18:1323-1329, 1975.

S. W. Churchill and M. Bernstein, "A Correlating Equation for Forced Convection From Gases and Liquids to a Circular Cylinder in Crossflow," Int. J. Heat. Mass. Tran. Trans. ASME 99, 1977, pp. 300-306.

J. A. Duffie and W. A. Beckman, "Solar Radiation" in Solar Engineering of Thermal Processes, 4th. Ed., Hoboken, New Jersey: Wiley, 2013, pp. 12-20.

J. Edman, "Dynamic Modeling of a Central Receiver CSP sytem in Dymola," M.S. thesis, Dept. En. Sci., Lund Univ., Lund, Sweden, 2014.

B. D. Ehrhart and D. D. Gill, "Evaluation of Annual Efficiencies of High Temperature Central Receiver Concentrated Solar Power Plants With Thermal Energy Storage," Sandia Nat. Lab., Albuquerque, NM, Rep. SAND2013-5493, Jul. 2013.

R. Ferri, A. Cammi and D. Mazzei, "Molten salt mixture properties in RELAP5 code for thermodynamic solar applications," Int. J. Therm. Sci. vol 47, 2008, pp. 1676-1687.

R. Forristal, "Heat Transfer Analysis and Modeling of a Parabolic Trough Solar Receiver Implemented in Engineering Equation Solver," NREL, Golden, CO, Rep. NREL/TP550-34169, Oct. 2013.

T. Fujii, H. Uehara, "Laminar natural-convective heat transfer from the outer surface of a vertical cylinder," Int. J. Heat. Mass. Tran. 13:607-615, 1970.

GeoModel Solar, Typical Meteorological Year Data (Sample Data), GeoModel Solar, [Online], Available: http://geomodelsolar.eu/data/typical-meteorological-year, [Accessed: Feb. 2014]. 
R. W. Haywood, "Advanced steam-turbine plant" in Analysis of Engineering Cycles, 4th. Ed., Pergamon Press, 1991, pp. 110-115.

J. E. Pacheco, "Final Test and Evaluation Results from the Solar Two Project," Sandia Nat. Lab., Albuquerque, NM, Rep. SAND2002-0120, Jan. 2002.

M. J. Reno, C. W. Hansen and J. S. Stein, "Global Horizontal Irradiance Clear Sky Models: Implementation and Analysis," Sandia Nat. Lab., Albuquerque, NM, Rep. SAND20122389, Mar. 2012.

M. Thern, Lund University, F. Eng., Dept. En. Sci., private communication, Dec 2013. 